\title{
IN-SITU, DYNAMIC MAGNETO-OPTICAL STUDIES OF THE GROWTH OF Co/Pt MULTILAYERS
}

\author{
R Atkinson and W R Hendren \\ Department of Pure and Applied Physics, The Queen's University of Belfast, BELFAST, BT7 INN, UK
}

\begin{abstract}
High precision, in-situ measurements of the magneto-optical polar Kerr rotation have been made continuously during the deposition of a series of $\mathrm{Co}$ and $\mathrm{Pt}$ multiple layers deposited by d.c. magnetron sputtering. Induced inhomogeneous magnetic moments in the $\mathrm{Pt}$ have been clearly observed and shown to fit very well to an exponentially decaying spatial function. The measured variations of Kerr rotation throughout the deposition of the Co and Pt layers can be simulated by classical electromagnetic theory using the optical and magneto-optical constants for $\mathrm{Co}$ and $\mathrm{Pt}$. The latter are deduced from independent ex-situ measurements on several $\mathrm{Co} / \mathrm{Pt}$ multilayers.
\end{abstract}

KEYWORDS: IN-SITU MAGNETO-OPTICS, MULTILAYERS, CO/PT, VOIGT PARAMETERS, KERR ROTATION.

\section{INTRODUCTION}

Alloys of Co and Pt, both in bulk and thin film form have been of technological interest for many years. More recently however, it has been discovered that ultra-thin multilayered structures of $\mathrm{Co}$ and $\mathrm{Pt}$ exhibit strong perpendicular magnetic anisotropy and enhanced magneto-optical activity, particularly below spectral wavelengths of the order of $450 \mathrm{~nm}$. These two material characteristics, combined with good chemical stability, make multilayered $\mathrm{Co} / \mathrm{Pt}$ an attractive medium for highdensity magneto-optic recording.

In any magneto-optically active medium, the origins of the magneto-optic effects and the possibility of their enhancement [1] are of considerable interest, both from fundamental and technological points of view. This is certainly the case for $\mathrm{Co} / \mathrm{Pt}$ and a considerable amount of work has already been carried out [2]. One of the most interesting features of $\mathrm{Co} / \mathrm{Pt}$ multilayers is the enhanced magneto-optic activity at short wavelengths, which exceeds that expected on the basis of the contribution due to Co atoms alone. For some time it has been realised that the increased activity is due to the induced magnetic moments associated with the $\mathrm{Pt}$ due to the close proximity of $\mathrm{Co}$ atoms [3]. Studies on bulk Co/Pt alloys suggest a possible $P t$ moment $\mu_{\mathrm{Pt}}$ of between $0.3-0.4 \mu_{\mathrm{B}}$, where $\mu_{\mathrm{B}}$ is one Bohr magneton. This is considerably less than that of $\operatorname{Co}\left(1.7 \mu_{\mathrm{B}}\right)$ and, though it has only a small effect on the magnetisation of the multilayer as a whole, it does have a profound effect on the magnetooptical activity. Moog et al [4] attempted to explain the unusually high Kerr effects observed in Co/Pt multilayers by introducing an effective Voigt parameter $\left(\mathrm{Q}_{\mathrm{P}}\right)[5]$ for the Pt layers, though in the model this was assumed to be real, rather than complex. With this simple expedient they found that they could explain their data if $\mathrm{Q}_{\mathrm{Pt}}$ was quite large, especially for wavelengths around $300 \mathrm{~nm}$. They pointed out that this was a surprising result considering that the magnetic moment for $\mathrm{Co}$ far exceeded that induced in Pt. More recently it has been shown, as one might expect, that the induced moment of the $\mathrm{Pt}$ decreases monotonically with distance from an interface with Co atoms. Rüegg et al [6] have presented both theoretical and experimental results, using $X$-ray absorption, which indicate that $\mu_{\mathrm{Pt}}$ varied with distance $d_{\mathrm{pt}}$ according to an inverse relationship with $\mu_{\mathrm{Pt}}=0.16 \mu_{\mathrm{B}}$ and $0.025 \mu_{\mathrm{B}}$ at $\mathrm{d}_{\mathrm{Pt}}=9 \AA$ and $40 \AA$ respectively.

In this paper we present the results of an in-situ experiment where we followed the temporal evolution of the magneto-optic polar Kerr effect during the sequential growth of several $\mathrm{Co}$ and $\mathrm{Pt}$ sputter deposited multiple layers. The resulting changes of Kerr rotation, both due to $\mathrm{Co}$ and polarised $\mathrm{Pt}$, are discussed and modelled using a magneto-optic theory capable of dealing with inhomogeneously magnetised media.

We demonstrate the applicability of classical electromagnetic theory for dealing with ultra-thin layers and determine the functional dependence of the magnetooptic parameter of polarised $\mathrm{Pt}$ atoms on distance from a plane interface with $\mathrm{Co}$.

\section{EXPERIMENTAL}

\section{Film Depositions}

In-situ observations were made with films deposited onto glass substrates using balanced d.c. magnetron sputtering at an argon pressure of $10^{-3}$ to $10^{-2} \mathrm{mbar}$. The target-to-substrate distance was $130 \mathrm{~mm}$ and deposition rates were $1 \AA / \mathrm{sec}$ and $0.3 \AA / \mathrm{sec}$ for $\mathrm{Pt}$ and $\mathrm{Co}$, respectively. Each magnetron was shuttered separately by manually operated shutters. These were opened and closed sequentially with an interval of a few minutes between successive layers. A quartz-crystal was employed to supplement film thickness monitoring which was determined by a timing procedure. 


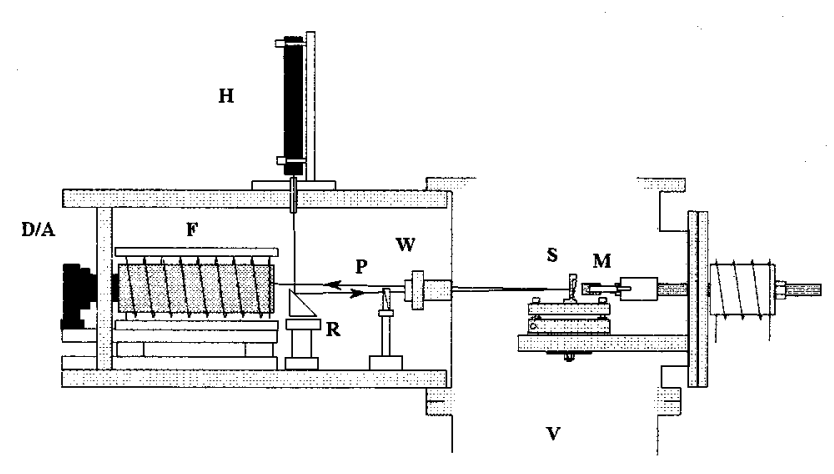

Figure 1. In-Situ Kerr Polarimeter. H: HeNe laser; R: Reflector; P: Polariser; W: Window; S: Sample; M: Magnet; F: Faraday modulator; A: Analyser; D: Detector; V: Vacuum Chamber.

\section{Magneto-optic Monitoring}

A stainless steel chamber contained two $50 \mathrm{~mm}$ dia, magnetrons, sample holder, quartz crystal monitor, and ion beam cleaning facility. Film growth monitoring facilities included a fast automatic ellipsometer and a normal incidence Kerr polarimeter. Both instruments operated with radiation of wavelength $633 \mathrm{~nm}$. The Kerr polarimeter, forming the main monitoring system relevant to the data to be presented here, is shown schematically in figure 1 together with the kinematic sample holder assembly and magnetic field switching arrangement.

Radiation from a stabilised, $10 \mathrm{~mW}, \mathrm{HeNe}$ laser is passed through a primary polariser and enters the work chamber through a low strain birefringence window. The beam is incident on the sample at an angle of incidence of less than $0.5 \mathrm{deg}$. After reflection, the radiation passes through a Faraday modulator followed by an analysing prism and finally a solid state detector. The Faraday modulator consists of a solenoid with a water-filled core. The windings of the solenoid are driven by a.c. for modulation purposes and also, simultaneously by d.c. for automatic nulling of the fundamental frequency components in the detected signal. A null occurs when the analyser extinction axis is coincident with the plane of polarisation of the reflected radiation (or the orientation of the major axis of the ellipse when elliptically polarised radiation is produced). The output from the detector is processed by a lock-in amplifier referenced to the a.c. modulation current in the solenoid. Any out-of-balance signal results in a d.c. output from the lock-in amplifier that is automatically integrated and coupled, through a power amplifier, to the solenoid windings. The integrated d.c. varies until a stable null setting is obtained.

Changes in the angle of the plane of polarisation of the reflected radiation immediately cause on out-ofbalance signal which is compensated for by an increase or decrease in the d.c. supplied to the Faraday coils. This current is therefore directly proportional to the rotation produced by the M.O. Kerr effect and can be calibrated directly.

The use of Faraday modulator techniques for ellipsometric nulling is well known and a full description of the construction of the Faraday cells can be found elsewhere [7]. It is sufficient to point out that the technique is one of the most precise methods for measuring small Kerr rotations with a sensitivity of better than \pm 1 arc sec.

\section{Voigt Parameter for Platinum}

In order to carryout any serious investigation of the evolution of magneto-optic activity in a growing $\mathrm{Co} / \mathrm{Pt}$ multilayer it is necessary to have a knowledge of the Voigt parameters $Q_{C_{o}}$ and $Q_{P t}$ for the two materials in ultra-thin film form. In the case of $\mathrm{Co}$, such parameters are available for the bulk form and these have been shown [8] to be reasonably applicable in the case of ultra-thin films. On the other hand the equivalent parameter for $\mathrm{Pt}$ is zero for the bulk but is finite for ultrathin films where there is an induced moment.

In order to determine $\mathrm{Q}_{\mathrm{Pt}}$ we initially performed a series of measurements on a set of $\mathrm{Co} / \mathrm{Pt}$ multilayers with equal thickness $\left(\mathrm{d}_{\mathrm{Co}}\right.$ and $\left.\mathrm{d}_{\mathrm{Ft}}\right)$ for the $\mathrm{Co}_{\mathrm{O}}$ and $\mathrm{Pt}$ that varied from $2.5 \AA$ to $30 \AA$. A single specimen was also measured having $\mathrm{d}_{\mathrm{Co}}=6.5 \AA$ and $\mathrm{d}_{\mathrm{Pt}}=18.5 \AA$.

The reason that equal thicknesses of $\mathrm{Co}$ and $\mathrm{Pt}$ where chosen was related to the concept of the single layer equivalence of multilayer structures. It has been shown earlier [9] that for optically absorbing media the effective Voigt parameter $\mathrm{Q}_{m}$ and complex refractive index $n_{m}$ of a multilayer may be related to those of the individual sub-layers by the expression

$$
Q_{m} n_{m}^{2} d_{m}=Q_{C o} n_{C o}^{2} d_{C o}+Q_{P t} n_{P t}^{2} d_{P t}
$$

where

$$
\mathrm{n}_{\mathrm{m}}^{2} \mathrm{~d}_{\mathrm{m}}=\mathrm{n}_{\mathrm{Co}}^{2} \mathrm{~d}_{\mathrm{Co}}+\mathbf{n}_{\mathrm{Pt}}^{2} \mathrm{~d}_{\mathrm{Pt}}
$$

and

$$
\mathrm{d}_{\mathrm{m},}=\mathrm{d}_{\mathrm{Co}}+\mathrm{d}_{\mathrm{Pt}}
$$

Clearly, where the ratio $\mathrm{d}_{\mathrm{Co}} / \mathrm{d}_{\mathrm{Pt}}$, and the sub-layer parameters are constants, the refractive index and $Q$ parameter of the multilayer as a whole should also be constant. In the case of refractive index, the values for $\mathrm{Co}$ and $\mathrm{Pt}$ are similar and consequently the effective refractive index for multilayers is not a strong function of $\mathrm{d}_{\mathrm{Co}}$ and $\mathrm{d}_{\mathrm{Pt}}$. On the other hand the Voigt parameter is very sensitive to changes the sub-layer thicknesses [10].

We carried out a comprehensive set of ellipsometric measurements on a number of $\mathrm{Co} / \mathrm{Pt}$ multilayers to determine the effective refractive indices of the films. In each case, total film thicknesses were in excess of $900 \AA$ 

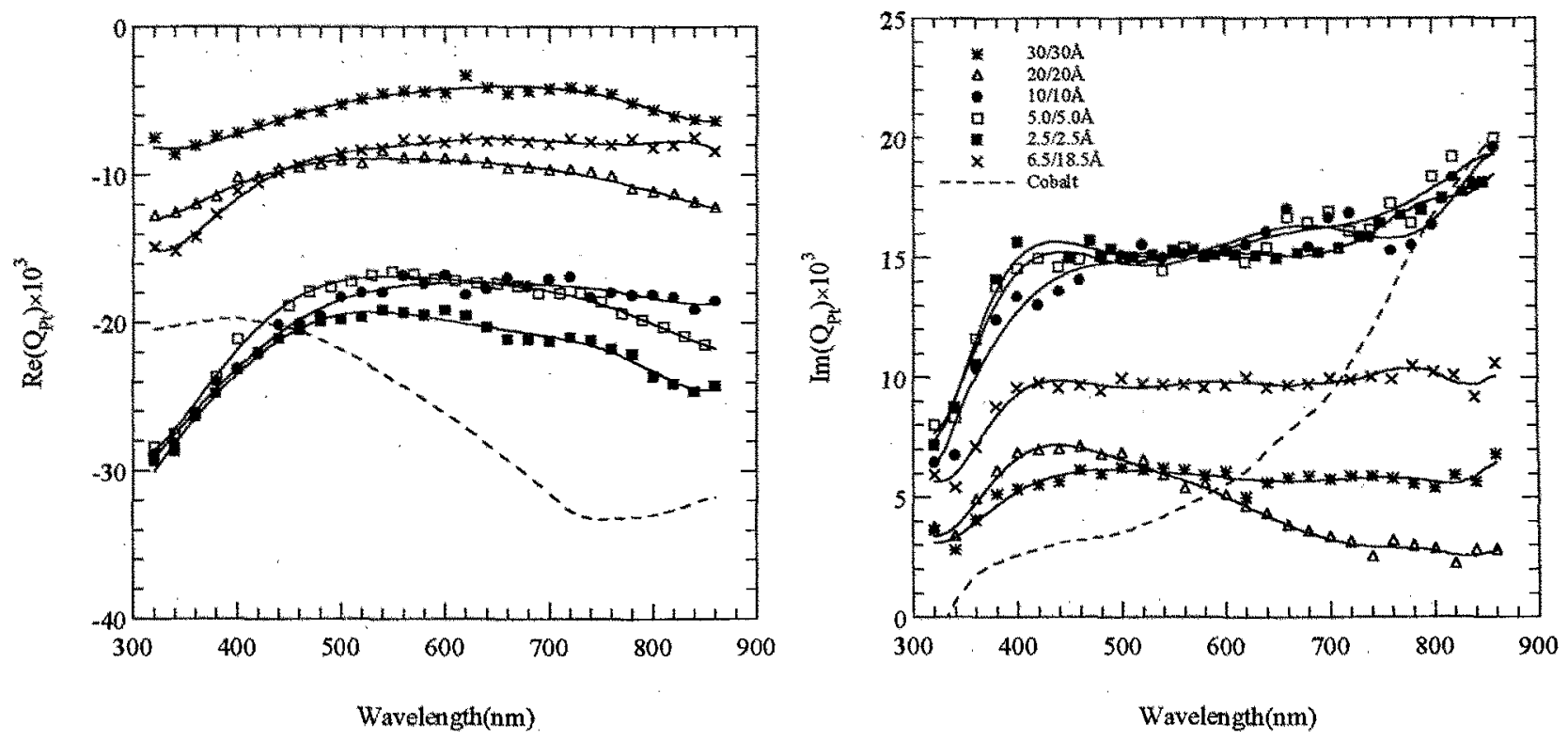

Figure 2. The complex Voigt parameter of Pt extracted form data on $\mathrm{Co} / \mathrm{Pt}$ multilayers

and therefore films were optically opaque. These were then combined with normal incidence measurements of the complex polar Kerr effect to determine the effective Voigt parameter. Again, since the films were opaque the complex Kerr rotation $\hat{\theta}$ is given by

$$
\hat{\theta}=\theta_{\mathrm{k}}+i \varepsilon_{\mathrm{k}}=\frac{i \mathrm{n}_{\mathrm{m}} \mathrm{Q}_{\mathrm{m}}}{\left(\mathrm{n}_{\mathrm{m}}^{2}-1\right)}
$$

where $\theta_{k}$ and $\varepsilon_{k}$ are the Kerr rotation and ellipticity respectively. It should be noted that a preferred sign convention was adopted as described elsewhere [11]. Having determined the dispersion of $\mathbf{n}_{\mathrm{m}}$ from ellipsometry, in the wavelength range $300-900 \mathrm{~nm}$, and the associated $Q_{\text {ru }}$ values through equation 4 it was possible using equations 1-3 to extract the Q-values for $\mathrm{Pt}$ assuming literature values for $\mathrm{n}_{\mathrm{Co}}, \mathrm{n}_{\mathrm{Pt}}$ and $\mathrm{Q}_{\mathrm{Co}}$ [12].

The results of these calculations are summarised in figure 2. For comparison we also show $\mathrm{Q}_{\mathrm{Co}}$ on the same figure. There are a number of points of interest concerning this figure which should be noted. First, in the determination of $\mathrm{Q}_{\mathrm{Pt}}$ it was assumed that the Pt layer had homogeneous magnetisation $\left(\mathrm{M}_{\mathrm{Pt}}\right)$ and therefore thickness independent $Q_{p t}$. Second, it is immediately obvious that $\mathrm{Q}_{p t}$ is finite and significant compared to $\mathrm{Q}_{\mathrm{co}}$. Third, there are large variations with wavelength but more importantly with sub-layer thickness values. This latter point is important and significant in understanding the thickness dependence of the $\mathrm{Qpt}_{\mathrm{pt}}$ parameter.

Returning to equations $1-3$ we expect that, on the basis of the above assumption (homogeneous $\mathrm{M}_{\mathrm{Pt}}$ ) and the constant ratio $\mathrm{d}_{C /} / \mathrm{d}_{\mathrm{Pt}}$, the $\mathrm{Q}_{\mathrm{pt}}$ values for this set of films would be very similar to each other. In fact this is not the case. Where $d_{p t}>10 \AA$ the $Q_{p t}$ values gradually decrease. The reason for this is quite obvious and is explained by the increasing proportion of non-polarised $\mathrm{Pt}$ in the multilayer. Such an observation is also consistent with the $Q_{p t}$ values for the single $\mathrm{Co} / \mathrm{Pt}$ $(6.5 / 18.5 \AA)$ multilayer where, again small values of $\mathrm{Q}_{\mathrm{ft}}$ are obtained.

In contrast, where $d_{p t}<10 \AA$ the $Q_{p t}$ values are all very similar and more importantly, appear to reach a maximum saturated value. We therefore conclude that within these multilayers the maximum induced moment on the $\mathrm{Pt}$ atoms is found. It is for this reason that we adopt these values of $\mathrm{Q}_{\mathrm{pt}}$ as being applicable to situations where the Pt is fully polarised with maximum moment at room temperature. Consequently, we use these in subsequent simulations of in-situ magneto-optical observations of growing $\mathrm{Co} / \mathrm{Pt}$ multilayers. For information, we present particular values of $\mathrm{n}$ and $\mathrm{Q}$ for both $\mathrm{Co}$ and $\mathrm{Pt}$ at a wavelength of $633 \mathrm{~nm}$ in table (I).

Table I Optical and Magneto-optical constants of Co and Pt $(\lambda=633 \mathrm{~nm})$

\begin{tabular}{|c|c|c|}
\hline Material & $\mathbf{n}$ & $\mathrm{Q} \times 10^{3}$ \\
\hline $\mathrm{Co}$ & $2.75+\mathrm{i} 4.53$ & $-27.4+\mathrm{i} 6.86$ \\
$\mathrm{Pt}$ & $2.30+\mathrm{i} 5.29$ & $-19.0+\mathrm{i} 15.0$ \\
Glass & $1.50+\mathrm{i} 0.00$ & 0 \\
\hline
\end{tabular}




\section{Dynamic In-situ Observations}

A study was made of the dynamic growth of several $\mathrm{Co} / \mathrm{Pt}$ sequences deposited, in each case on a $200 \AA \mathrm{Pt}$ buffer layer on glass. The Kerr rotation was monitored continuously in an applied perpendicular field of $0.2 \mathrm{~T}$. To determine, and subsequently correct, any instrumental angular drift, the sample magnetisation was temporarily reversed every 2 secs. In general, however, any drift in the azimuthal zero point was negligible.

Ideally, when looking for the effects of induced moments in $\mathrm{Pt}$ one would like to simplify the experimental arrangements by referring to a single boundary, say between $\mathrm{Co}$ and $\mathrm{Pt}$. This might be done using an optically opaque Co layer. However, because of the very large fields required to saturate such a film in the perpendicular direction this is impractical. Instead, it is necessary to grow simple multilayered structures where the Co layer is thin enough to favour perpendicular anisotropy. Consequently, ultra-thin $(\leq 5 \AA)$ Co layers were deposited on top of the Pt buffer layer and subsequent Pt layers.

Magneto-optically derived hysteresis loops were examined, ex-situ, after the deposition was completed. A number of deposition sequences were observed which illustrated some interesting features. These are enumerated below together with a discussion of the main points.

1. Figure 3 illustrates the growth of the structure $\operatorname{Pt}(200 \AA)) / 2 x[\operatorname{Co}(5 \AA) / \operatorname{Pt}(9 \AA)][\operatorname{Co}(5 \AA)]$. First, it is observed that no Kerr rotation is observed in the $\mathrm{Co}$ until the layer is between 1-2 $\AA$ thick. Second, it is clear that the deposition of $\mathrm{Co}$ leads to an increase in Kerr rotation which levels off after about $3 \AA$ in the first layer. In subsequent $C o$ layers the rotation appears to reach a maximum at about $3 \AA$ and then begins to decrease. It is believed that this decrease is the result of lost perpendicular anisotropy in the last few angstroms of the Co layer and consequently the low $0.2 \mathrm{~T}$ field cannot pull the magnetisation of this section of film out of the plane. However, at the start of the Pt deposition the anisotropy returns immediately and the rotations actually begin at

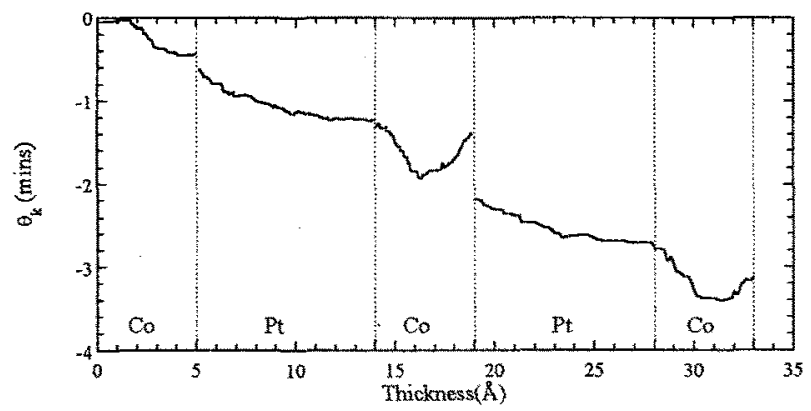

Figure 3. Continuous monitoring of the deposition of a $\operatorname{Co}(5 A) / \mathrm{Pt}(9 \AA)$ system on $200 \AA$ Pt buffer layer. points where extrapolation of the earlier parts of the Cocurves would suggest they might. It was observed that this return of perpendicular anisotropy could be triggered by the Pt atoms or by excessive exposure to the residual gases of the vacuum chamber. It is worth pointing out that no discreet jump in Kerr rotation is observed when, $\mathrm{Co}_{0}$ is added to the $\mathrm{Pt}$. This demonstrates the integrity and stability of the experimental system. Third, it is quite clear that the deposition of the $\mathrm{Pt}$ further increases the Kerr rotation and that the increase shows a levelling off beyond $\mathrm{d}_{\mathrm{Pt}} \approx 7 \AA$.

Finally, the hysteresis loop for the structure, on its removal from the vacuum system, was square and of low coercivity demonstrating that the majority of material within the structure has large perpendicular anisotropy.

2. Figure 4 shows the evolution of the sequential deposition of $\operatorname{Pt}(200 \AA) / 3 \mathrm{x}[\mathrm{Co}(2 \AA) / \operatorname{Pt}(30 \AA)]$. At first sight this deposition sequence seems uninteresting. However, it does illustrate some important features. As was found in the previous case, Co layers less than $2 \AA$ show little or no increase in Kerr rotation. Where sudden increases are seen, these rapidly decay away. This may seem reasonable until one examines the $\mathrm{Pt}$ depositions. In two cases, the addition of $\mathrm{Pt}$ immediately produced a rapid increase in Kerr rotation that reaches a maximum

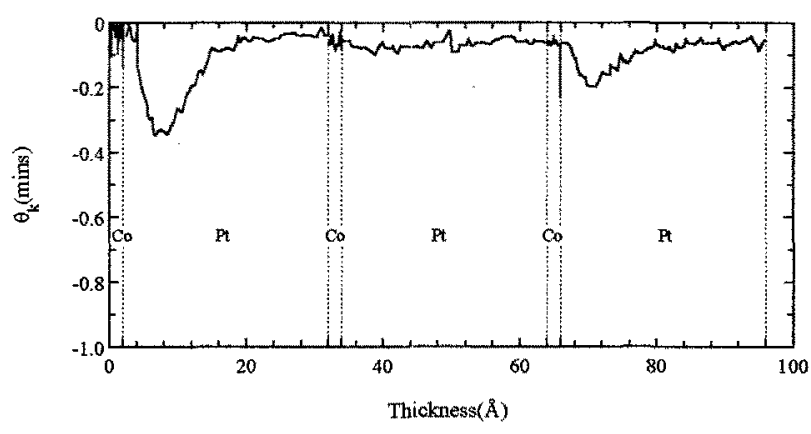

Figure 4. Continuous monitoring of the deposition of a $\operatorname{Co}(2 \AA) / \operatorname{Pt}(30 \AA)$ system on $200 \AA \mathrm{Pt}$ buffer layer.

for $d_{\mathrm{Pt}} \simeq 7 \AA$. Thereafter the rotation decays away, due to decreasing moment and the absorbing properties of the Pt. The middle period does not show this effect, presumably because of poor control of the very low $\mathrm{Co}$ thickness. The most interesting, and perplexing, feature of this monitoring sequence is the total lack of rotation caused by the $\mathrm{Co}$ layer. One may argue from the experimental evidence that the $\mathrm{Co}$ induces a moment in the following $\mathrm{Pt}$ layer since the result is seen quite spectacularly. However, the question of why the Co does not induce a similar moment in the underlying $\mathrm{Pt}$ layer remains unanswered. 


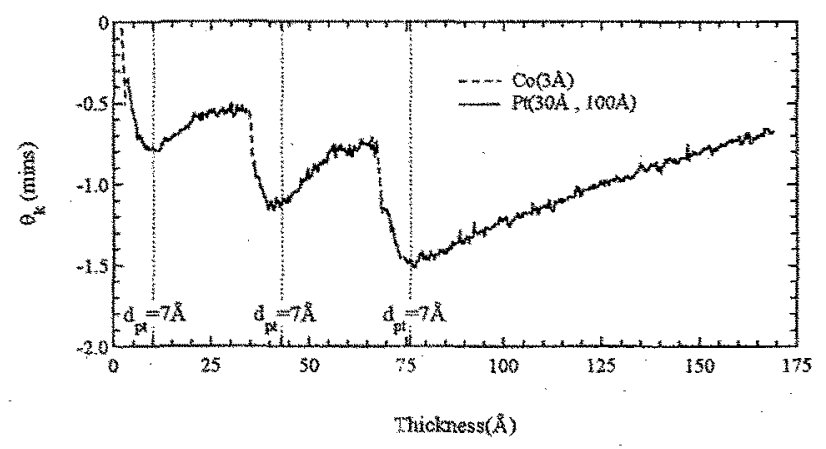

Figure 5. Continuous monitoring of the deposition of a $2 x[\operatorname{Co}(3 A) / P(30 A)] / C o(3 A) / P t(100 A)$ system on 200 A Pt buffer layer.

3. In the final sequence, $\operatorname{Pt}(200) \AA / 2 x[\operatorname{Co}(3 \AA) / \operatorname{Pt}(30 \AA)]$ $/ \mathrm{Co}(3 \AA) / \mathrm{Pt}(100 \AA)$ we see very clearly, in figure 5 the stepped increase in Kerr rotation as each successive layer is added to the system. Each time the Co layer is added one observes an increase of Kerr rotation of about 0.5 mins. Again careful examination of the curves reveals the delayed response until the critical thickness of Co of approximately 1-2 $\AA$ is reached. This is then followed by each Pt layer resulting in a further increase which again peaks at 0.4-0.5 mins for $d_{\mathrm{Pt}} \sim 7 \AA$. Beyond this there is a decrease in the rotation which is most vividly illustrated by the last $\mathrm{Pt}$ layer deposited to a thickness of $100 \AA$. This curve demonstrates quite clearly the gradual evolution of the magneto-optical activity of a $\mathrm{Co} / \mathrm{Pt}$ multilayer and provides unequivocal evidence, as do the other curves, for the induced magnetic moment of the Pt layer.

\section{DISCUSSION/MODELLING}

Having established the experimental curves showing the developing Kerr rotation of $\mathrm{Co} / \mathrm{Pt}$ multiple layers it is useful to examine these in a more quantitative way using calculations based on classical electromagnetic theory. In order to carry out such calculations it must be remembered that the induced moment of the $\mathrm{Pt}$ atoms is expected to be a function of distance $\mathrm{z}$ from the Co-Pt interface. Consequently, the Voigt parameter for Pt must be written $Q_{p t}=Q_{0} f(z)$ where $f(z)$ defines the spatial variation of induced magnetic moment and $Q_{o}$ is the saturated value deduced earlier and given in table $I$. The calculation of the complex Kerr rotation for the growing film may be performed in three ways. One may apply, the [4x4] matrix approach [13] for M.O. multilayer calculations where each layer of $\mathrm{Pt}$ is sub-divided into many microscopic $(\ll 1 \AA)$ layers, each assumed to be homogeneous with a Q-value determined by the spatial function $f(z)$ in accordance with the position of the microscopic sub-layer within the Pt. Alternatively, one may use a modified [4x4] matrix approach that specifically deals with inhomogeneous systems. Such an approach has recently been discussed in detail by
Atkinson and Kubrakov [14]. A third technique and one which is convenient here, is based on the differential reflectance approach [15] in combination with the principle of superposition of magneto-optic effects [16]. This states that the magneto-optic Kerr coefficient $(k)$ for any system is a linear superposition of the elemental Kerr coefficient (dk) for individual layers, where each $\mathrm{dk}$ value is evaluated assuming the rest of the system is nonmagnetic. If we make the assumption that the isotropic refractive index of the $\mathrm{Co} / \mathrm{Pt}$ system is homogeneous, which is reasonable here since the structures are mostly $\mathrm{Pt}$, then the complex Kerr coefficient for a film of finite total thickness $d$ is given by

$$
\mathrm{k}=\int_{0}^{\mathrm{d}} \mathrm{B}_{0} \mathrm{q}(\mathrm{z})\left[1+\mathrm{B}_{1} \mathrm{e}^{-2 \alpha z}+\mathrm{B}_{2} \mathrm{e}^{-\alpha z}\right] \alpha \mathrm{e}^{\alpha z} \mathrm{dz},
$$

where $\quad B_{0}=\mathrm{in}_{\mathrm{m}} /\left(\left(1+\mathrm{n}_{\mathrm{m}}\right)\left(1+\mathrm{r}_{\mathrm{o}} \mathrm{r}_{\mathrm{s}} \mathrm{e}^{\alpha \mathrm{d}_{\mathrm{o}}}\right)\right)^{2}$,

$$
B_{1}=r_{s}^{2} e^{2 \alpha d}, \quad B_{2}=2 r_{s} e^{\alpha d},
$$

and

$$
\alpha=14 \pi n_{m} / \lambda .
$$

$r_{0}$ and $r_{3}$ are the Fresnel amplitude reflection coefficients at the top and bottom interfaces of the film, given by $r_{0}=\left(1-n_{m}\right) /\left(1+n_{m}\right)$ and $r_{s}=\left(n_{n}-n_{s}\right) /\left(n_{m}+n_{s}\right)$ where $n_{s}$ is the refractive index of the substrate. $q(z)$ is a ID spatial function describing the variation of $Q$ through the whole multilayer structure. This will be a constant equal to $Q_{c o}$ in the $C_{0}$ layer and will be equal to $Q_{0} f(z)$ in the Pt layers.

Once $\mathrm{k}$ has been determined by either of these methods one can determine the amplitude reflectance $r$ using the well known reiterative reflectance formula and then form the complex Kerr rotation $\hat{\theta}_{k}(=\mathbf{k} / \mathbf{r}$, where $\mathrm{k}<<\mathrm{r}$ ). The integral method, with its assumptions of constant refractive index, is by far the simplest and fastest technique of calculation and, for these materials, gives, more or less, identical results to the rigorous calculations.

In figure 6 we show an example of a magnified section of the growth curve corresponding to the deposition of $\operatorname{Pt}(200 \AA) / \mathrm{Co}(3 \AA) / \mathrm{Pt}(30 \AA)$. This curve is typical of the form generally observed and corresponds to the maximum changes in rotation that we observed experimentally. In modelling this curve we have two options. We may consider the effect of the Co layer and ignore the polarising effect that such a layer may have on the underlying $200 \AA \mathrm{Pt}$ layer; or, we may include the effects of the polarisation of the Pt in the calculations. If we choose the latter, which in the first instance seems reasonable, we find that the resulting rotations are too large since they represent the M.O. activity of both the 


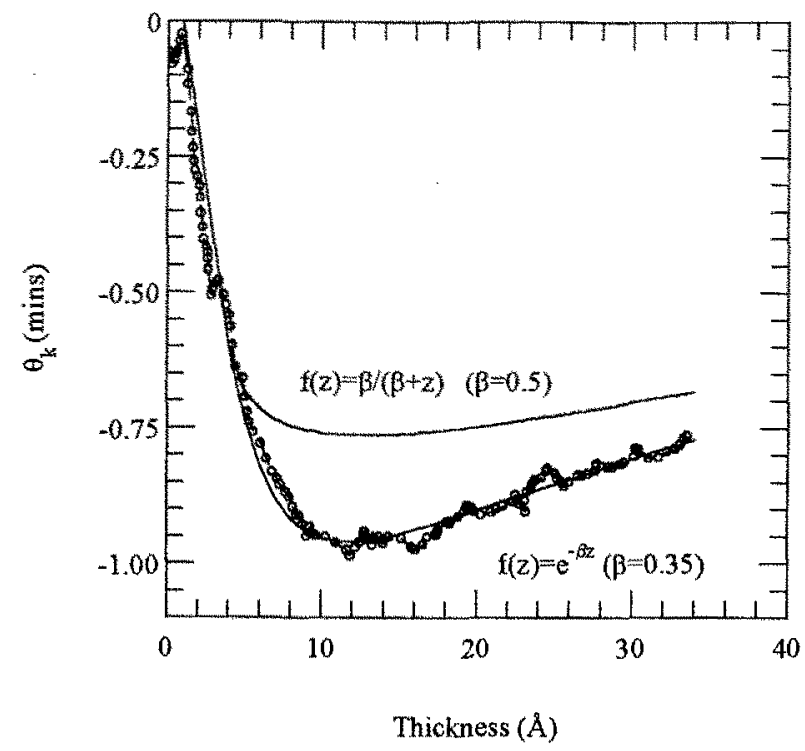

Figure. 6. Theoretical fit to monitored Kerr rotation for a $\operatorname{Co3} \mathrm{A} / \mathrm{Pt} 30 \mathrm{~A}$ on Pi200A buffer layer.

Co and the underlying polarised $\mathrm{Pt}$ layer. However, as already mentioned we have experimental evidence from the work dealing with the $2 \AA$ Co layers which certainly indicate the ineffectiveness of $\mathrm{Co}$ to polarise the $\mathrm{Pt}$ atoms upon which they are deposited. The reason for this is unclear but is supported by simulations of the growth curve for figure 6 where we only consider the M.O. activity of the Co layer and that of the Pt layer deposited on top of the Co.

Thus, in figure 6 , we show a theoretical curve corresponding to the deposition of $3 \AA$ of $\mathrm{Co}$ on top of $200 \AA$ of non-magnetic $\mathrm{Pt}$ followed by $30 \AA$ of $\mathrm{Pt}$ whose $\mathrm{Q}$-parameter is given by $\mathrm{Q}=\mathrm{Q}_{\mathrm{o}} \mathrm{e}^{-\beta z}$ where $\mathrm{z}\left(=\mathrm{d}_{\mathrm{Pt}}(\AA)\right)$ is the distance through the $\mathrm{Pt}$ layer from the interface with $\mathrm{Co} . \beta$ is the only fitting parameter found to be equal to 0.35 . For comparison we also tried to fit the curve using the inverse relationship $Q=Q_{0} \beta /\left(d_{P t}+\beta\right)$. This was found to be inadequate. A typical curve for $\beta=0.5$ is shown in figure 6.

\section{CONCLUSIONS}

Kerr rotations have been measured, contimuously, insitu during the sequential deposition of a series of $\mathrm{Co}$ and Pt multiple layers on a $\mathrm{Pt}(200 \AA)$ buffer layer on glass substrates. It was observed that, for Co layers less than $2 \AA$ thick, there was little or no Kerr effect, though considerable M.O. activity could be induced in a following Pt layer. With Co layer thicknesses $\geq 3 \AA$ good perpendicular anisotropy was observed and Kerr rotations were consistent with those predicted on the basis of the bulk constants for Co. It was unnecessary to invoke the idea of an induced moment in the underlying $\mathrm{Pt}$ layer. The reason for the absence of magnetic moment in the Pt underlayer is problematic and needs further investigation. However, we have presented both experimental and theoretical evidence for this phenomenon in our films. It is our opinion that the effect may be related to the time interval between the deposition of the $\mathrm{Pt}$ and $\mathrm{Co}$ layers, during which there may be some small interfacial contamination of the $\mathrm{Pt}$ surface that inhibits the ability of the $\mathrm{Co}$ to induce moments in the underlying $P t$. We do have some ellipsometric evidence to support such a statement though, due to limitation of space, we cannot include this here.

Direct evidence for induced moments in the Pt layer deposited on top of the Co is seen clearly and is reproducible. Moreover, by treating such layers as inhomogeneous with a magneto-optic parameter given by $\mathrm{Q}_{\mathrm{Pt}}=\mathrm{Q}_{0} \mathrm{e}^{-\beta z}$, with $\beta=0.35$, we have been able to fit the experimentally observed variations in Kerr rotation remarkably well indeed.

\section{REFERENCES}

[1] R Atkinson, J Magn Magn Mat, 124, 178 (1993)

[2] W B Zeper, F I A M Greidanus and P F Carcia, IEEE Trans. Magn, 25,3764 (1989)

[3] T R McGuire, J A Aboaf and E Klokholm, J. Appl. Phys., 55, 1951 (1984)

[4] E R Moog, J Zak and S. Bader, J. Appl. Phys 69 , $880(1991)$

[5] R Atkinson, R Gamble, G P Gu, PH Lissberger, Thin Solid Films, 162,89 (1988)

[6] S Rüegg, G Schütz, P Fischer, R Wienke, W B Zeper and H Ebert, J. Appl. Phys., 691951 (1984)

[7] P H Lissberger, I W Salter, M Fitzpatrick and P L Taylor, J. Phys. E., 10, 635,(1997)

[8] R Atkinson, W R Hendren, I W Salter, M J Walker, J Magn Magn Mat, 129, 442 (1994)

[9] R Atkinson, J Mag and Magn Mat, 95, 61(1991)

[10] Meng, W. P. van Drent, J.C. Lodder, and Th. J. A. Popma to be published in J. Magn. Magn. Mater. (1996)

[11] R Atkinson, P H Lissberger, App Optics, 31, 6076 (1992)

[12] R Atkinson, J. Magn. Magn. Mater. 124, 333 (1993

[13] Zhan-Ming $\mathrm{Li}, \mathrm{B} \mathrm{T}$ Sullivan and $\mathrm{R} \mathrm{R}$ Parsons, Appl Opt, 27, 1334(1988)

[14] R Atkinson and N K Kubrakov, Proc of Royal Soc A, 449, 205 (1995)

[15] R Atkinson, J Magn Magn Mat, 115, 353 (1992)

[16] R Atkinson and PH Lissberger, I Magn Magn Mat, 118,271 (1993) 